\title{
Ángel Rama y el ensayo cosmopolita
}

\author{
Alejandra Josiowicz \\ Rutgers University, Estados Unidos.
}

\section{Resumen}

Aunque los proyectos ensayísticos más extensos de Ángel Rama han sido profusamente discutidos y analizados, menos atención se ha concedido a su constante dedicación a los ensayos de mediana o breve extensión. El ensayismo mediano y breve de Rama -en una relación a la vez continua y paradójica con los sistemas interpretativos abarcadores de sus ensayos más extensos- tiende redes conceptuales provisorias, que responden a propósitos puntuales en torno a debates del ámbito cultural y literario. En este trabajo, analizo los ensayos académicos que Ángel Rama publicó entre 1981 y 1983, una etapa de exilio y dislocamiento geográfico, en los que examino la emergencia de una red conceptual que enlaza lo cosmopolita, lo internacional y lo transatlántico con una reflexión sobre la tecnificación cultural. En lugar de una única categoría o estructura hermenéutica, Rama rearma en cada ensayo la trama conceptual, creando constelaciones distintas, en un esfuerzo por ir más allá de un marco nacional o latinoamericanista para pensar la historia literaria y cultural.

Palabras clave: Ensayo, Ángel Rama, cosmopolitismo, latinoamericanismo, tecnificación cultural.

\begin{abstract}
Although Ángel Rama's lengthier essayistic projects have been profusely discussed and analyzed, his constant dedication to shorter and medium-length essay pieces has received remarkably less attention. Rama's shorter essays -in a continuous and paradoxical relation with the comprehensive interpretative systems of his more extended pieces- tend to create provisional conceptual linkages and aim to intervene in specific literary and cultural debates. This work analyzes Rama's academic essays published between 1981 and 1983, a period of exile and geographic displacement, in order to examine the emergence of a conceptual framework that links cosmopolitanism, internationalism and the transatlantic, together with a reflection on technology and culture. Instead of postulating a unique category or hermeneutic structure, Rama reassembles the conceptual map in each piece, in an effort to go beyond a national or latinamericanist frame to consider literary and cultural history.
\end{abstract}

Keywords: Essay, Ángel Rama, cosmopolitanism, latinamericanism, technology and culture. 
1. Como ejemplo, puede pensarse en la jerarquía espacial y simbólica de la ciudad letrada ante el resto de los discursos sociales (orales, flexibles, plebeyos), la transculturación como síntesis de lo modernizador-cosmopolita y el sustrato regional, o el boom como fenómeno de la sociedad de consumo, síntoma del nuevo dandismo del autor.

2. MARX, Karl y ENGELS, Friedrich. The Communist Manifesto, 2005.
Aunque los proyectos ensayísticos más extensos de Ángel Rama (1926-1983), esto es, Rubén Darío y el modernismo (1970), Transculturación narrativa en América Latina (1982), La ciudad letrada (1984), Las máscaras democráticas del modernismo (1985), o la edición de Más allá del Boom: Literatura y mercado (1981), han sido profusamente discutidos y analizados en aulas académicas y seminarios, menos atención se ha concedido a su constante dedicación a los ensayos de mediana o breve extensión. El ensayismo mediano y breve de Rama -dedicado a la observación puntual y detallada y a la intervención en polémicas concretas- es revelador de que, en una relación a la vez continua y paradójica con los sistemas interpretativos abarcadores de sus ensayos más extensos ${ }^{1}$, Rama tiende a trazar redes conceptuales provisorias, que responden a propósitos puntuales en torno a debates del ámbito cultural y literario. En este trabajo, analizo los ensayos académicos que Ángel Rama publicó entre 1981 y 1983 en Review (Americas Society), Nueva Sociedad, Hispamérica, Nueva Revista de Filología Hispánica y Revista de Crítica Literaria Latinoamericana, en una etapa de exilio y dislocamiento geográfico en vida del crítico, en los que examino la emergencia de una red conceptual que enlaza lo cosmopolita, lo internacional y lo transatlántico con una reflexión sobre la tecnificación cultural. En lugar de una única categoría o estructura hermenéutica, Rama rearma en cada ensayo la trama conceptual, creando constelaciones distintas, en un esfuerzo por ir más allá de un marco nacional o latinoamericanista para pensar la historia literaria y cultural.

\section{Hacia una internacional de las letras}

En su "Manifiesto comunista" de 1848, Marx y Engels afirman que una modalidad global de la producción y el consumo y una interdependencia universal de las naciones, cuyo carácter cosmopolita ha horadado las bases de las industrias nacionales, caracterizan tanto la producción material como la intelectual:

The intellectual creations of individual nations become common property. National one-sidedness and narrow-mindedness become more and more impossible, and from the numerous national and local literatures, there arises a world literature. ${ }^{2}$ 
Este proyecto marxiano de una internacional de la creación intelectual, así como de una literatura mundial que supere el marco estrecho de lo nacional es, como argumento en este ensayo, foco central del régimen cosmopolita de la modernidad letrada en Ángel Rama, que adquiere un lugar central en sus últimos ensayos, entre 1981 y 1983. Exiliado en Venezuela debido a la dictadura uruguaya, Ángel Rama vivió y trabajó desde 1979 en varias universidades de los Estados Unidos, hasta que el gobierno de ese país le negó la residencia permanente, por lo que se trasladó a París, falleciendo en 1983 en un accidente aéreo. En ese breve período, Ángel Rama escribió numerosos ensayos de tipo académico: "Founding the Latin American Literary Community" (1981), "La tecnificación narrativa" (1981), "USA y los escritores latinoamericanos” (1982), “Los ríos profundos, ópera de pobres” (1983), “La modernización literaria latinoamericana (1870-1910)” (1983), “José Martí en el eje de la modernización poética: Whitman, Lautréamont, Rimbaud” (1983), "Literatura y cultura en América Latina” (1983) y "De la concertación de los relojes atlánticos” publicado póstumamente en 1984. Si bien en cada uno de ellos la preocupación por los sistemas transnacionales aparece bajo modalidades diferentes, la recurrencia de la categoría de un internacionalismo de las letras y de la vida intelectual resulta reveladora de una tendencia comparatista que, si bien es deudora de la idea marxiana de una "internacional de las letras" y del internacionalismo comunista, va más allá, trazando flujos múltiples y sin centro fijo para la literatura mundial. ${ }^{3}$ El proyecto de una historia social de los contextos de producción, recepción y circulación internacional de las ideas, por otro lado, ligan a Rama con los planteamientos posteriores de Pierre Bourdieu, quien en su estudio del campo intelectual transnacional europeo, aspira a "desnacionalizar" las categorías de pensamiento como condición de un deseado universalismo intelectual ${ }^{4}$. Tal como más tarde Franco Moretti, Rama es lector de las teorías del sistema-mundo de Immanuel Wallerstein, y establece un paralelo entre la producción literaria y el capital económico global. Sin embargo, mientras la admiración de Moretti por la historia cuantitativa y el privilegio de un método científico tiende a la unión de biogenética y filología ${ }^{5}$, Rama privilegia el análisis histórico-cultural de las estructuras y técnicas de poder simbólico a través de las cuales la recepción y producción de la literatura mundial pueden abrir paso a formas de la alteridad. El proyecto de unir los estudios literarios con un mapa socio-económico pos-nacional no sólo le permite explorar las geografías móviles del exilio intelectual, la diáspora y la dispersión de los públicos lectores, sino que también contribuye a un comparatismo literario que rearma una cartografía intelectual global sin centro ni paradigma global hegemónico.
3. En el ámbito de los estudios cosmopolitas, las categorías conceptuales utilizadas son arena de intensos debates teóricos, dadas las implicaciones cívico-políticas y para el estatuto de la literatura comparada como disciplina. Conceptos como intraducibilidad, incomparabilidad, cosmopolítico o, en el caso de Gayatri Chakravorty Spivak, "planetariedad" (planetarity) constituyen intentos de abrir un sentido de alteridad ético-política al interior del comparatismo. Para una crítica del concepto de traducción y traducibilidad, puede verse el reciente y polémico Dictionary of Untranslatables: A Philosophical Lexicon (2014), traducido al inglés por Jacques Lezra, Emily Apter y Michael Wood, que contiene la entrada "Planetariedad", de Spivak, o el estudio de Emily Apter, Against World Literature. On the Politics of Untranslatability (2013). Para un análisis del cosmopolitismo en América Latina, también puede verse el estudio de Mariano Siskind, Cosmopolitan Desires: Global Modernity and World Literature in Latin America (2014).

4. BOURDIEU, Pierre.

"Les conditions sociales de la circulation internationale des idées". Actes de la recherche en sciences sociales, 2002, p. 8.

5. Cf. MORETTI, Franco.

"Conjectures on World

Literature". New Left Review, 2000, y Graphs, Maps and Trees. Abstract Models for a Literary Theory, 2005. Para una crítica de estas teorías, ver el capítulo: APTER, Emily. "Moretti's Literary World-Systems", 2013, p. 48 y ss. 
6. RAMA, Ángel. "Founding the Latin American Literary Community". Review: Literature and Arts of the Americas. Americas Society, 1981.

7. Ibídem, p. 12-13.

8. BOURDIEU, Pierre.

"Les conditions sociales de la circulation internationale des idées". Actes de la recherche en sciences sociales, 2002, p. 7.

9. RAMA, Ángel. "La tecnificación narrativa". Hispamérica, 1981.

10. BOURDIEU, Pierre.

"Les conditions sociales de la circulation internationale des idées". Actes de la recherche en sciences sociales, 2002, p. 3.

\section{El exilio intelectual}

El ensayo que inaugura este período de reflexión sobre el intelectual y la diáspora transnacional apareció en un número especial de la revista Review. Americas Society con el título "Founding the Latin American Literary Community". Allí, Rama adjudica al quiebre de las lealtades nacionales dado por las persecuciones de los regímenes militares del Cono Sur el cambio de perspectiva que sufren los intelectuales Latinoamericanos: su pensamiento se "universaliza", encuentran a su "otro" latinoamericano en el exilio (el trabajador migrante, el "otro" de la diáspora), se vuelve global, "macro-estructural", continental, y supera las miras más acotadas del ensayo de interpretación nacional. La comunidad intelectual transnacional resultante, según Rama, fundaría las bases de la comunidad literaria diaspórica del futuro ${ }^{7}$. Como el proyecto de internacionalización de la vida intelectual de Pierre Bourdieu, quien busca localizar los "malentendidos" estructurales dados por la pérdida de los contextos de producción de las ideas, Rama argumenta que los exilios políticos, las migraciones económicas y las diásporas político-culturales habrían contribuido a la emergencia de una interpretación global del continente, así como a la mejora de las comunicaciones transnacionales entre los países sin la interposición de campos culturales mediadores (Estados Unidos o Europa) $)^{8}$. Tanto en el proyecto internacionalista desnacionalizado de Rama como en el universalismo intelectual de Bourdieu, se podría señalar el carácter neutro, abstracto - carente de de especificidad cívica o lingüística- de la deseada comunidad exiliada, transnacional de las letras.

\section{La transnacional técnica}

"La tecnificación narrativa" es otro ensayo centrado en la pulsión intelectual transnacional, aunque aquí el tema aparece ligado a la cuestión de la técnica. Rama examina lo internacional en el campo de la economía, en un nuevo paralelo con Bourdieu, quien afirma que una perspectiva de importación-exportación de las ideas puede tener un efecto de ruptura beneficioso para una historia social del intercambio intelectual ${ }^{10}$. Lector de Immanuel Wallerstein (The Modern World-System. Capitalist Agriculture and the Origins of the European World Economy in the Sixteenth Century. New York: Academic Press, 1976), Stanley y Barbara Stein (The Colonial Heritage of Latin America. New York: Oxford Univ. Press, 1970), Albert Hirsch- 
man ("The Political Economy of Import-Substitution Industrialization in Latin America". In: A Bias for Hope. Essays on Development and Latin America. New Haven and London: Yale University Press, 1971), y los reportes de la Comisión Económica para América Latina (CEPAL), Rama traza un paralelismo a-sincrónico entre los campos económico y artístico. Ante las "frustraciones de las esperanzas" productivas en América Latina y el desencanto con las teorías de industrialización por sustitución de importaciones de fines de los años ' $70^{11}$, las técnicas literarias posibilitarían una modalidad no-hegemónica de internacionalización del trabajo intelectual, desligado de un "centro externo concreto"12. Desde el cosmopolitismo esteticista del modernismo de fines del Siglo XIX, pasando por el programa estético-político transnacional de las vanguardias, hasta el boom de traducciones en las editoriales Latinoamericanas de 1960, las técnicas literarias determinarían una relación productiva con el mercado intelectual cosmopolita. La obra y la figura de escritor de Julio Cortázar (1914-1984) emerge aquí como ejemplo paradigmático del modo en que la internacionalización de las técnicas literarias

...habrían constituido una suerte de mercado común de las letras, al que concurrirían con el mismo derecho tanto europeos como americanos, tanto asiáticos como africanos, manejando las mismas fuentes y los mismos inventos, creando sobre ellos otros nuevos que pasarían a integrar el acervo común, siendo por lo tanto influidos e influyendo a la vez de conformidad con la fuerza y originalidad de sus libres aportaciones. ${ }^{13}$

Este "mercado común de las letras", similar a la comunidad trasnacional de Bourdieu, es un espacio de producción y absorción neutro, desnacionalizado; allí, los intelectuales marginales disputan un lugar en la comunidad internacional que la producción industrial periférica, con sus dificultades e insuficiencias infraestructurales, no podría pretender ${ }^{14}$. Las técnicas estéticas, como "instrumentos universales y neutrales", habrían dado lugar a un progresivo internacionalismo de los escritores, y a la "libre incorporación de materiales"15. A la vez que las técnicas aparecen en este análisis como instrumentos de posicionamiento al interior de los campos de producción cultural -la concepción instrumental y antropológica de la tecnología a la que se refiere Heidegger ${ }^{16}$-, también constituyen modos radicales de revelar el carácter productivo -no meramente imitativo- del intelectual periférico. A diferencia de los flujos tecnológicos industriales, que ponen en escena la persistencia de las relaciones de dependencia, las técnicas literarias podrían resultar liberadoras, en tanto revelan la potencia creativa del intelectual Latinoamericano, con posibles implicancias
11. En "The Political Economy of Import-Substitution Industrialization in Latin America", Albert Hirschmann da cuenta de dicho desencanto, trata de refutar el supuesto agotamiento del modelo de sustitución de importaciones, e intenta abrir "avenidas para el continuo crecimiento industrial". The Quarterly Journal of Economics, 1968, p. 32.

12. RAMA, Ángel. "La tecnificación narrativa". Hispamérica, 1981, p. 44.

13. Ibídem, p. 45

14. Ibídem, p. 47.

15. Ibídem, p. 75.

16. HEIDEGGER, Martin. The Question Concerning Technology and Other Essays, 1977. 
17. Ibídem, p. 30-35.

18. RAMA, Ángel. “La modernización literaria latinoamericana". Hispamérica, 1983.

19. Ídem. "José Martí en el eje de la modernización poética: Whitman, Lautréamont, Rimbaud". Nueva Revista de Filología Hispánica, 1983.

20. Ídem. "La modernización literaria latinoamericana". Hispamérica, 1983, p. 15.

21. Ibídem, p. 18.

22. Ídem. "José Martí en el eje de la modernización poética: Whitman, Lautréamont, Rimbaud". Nueva Revista de Filología Hispánica, 1983, p. 117.

23. Ibídem, p. 127. para los campos no-estéticos de la producción. La técnica, en este sentido, aparece no como destino prefijado e ineludible de la modernidad cosmopolita, sino como espacio abierto de destinación ${ }^{17}$, en que el escritor se encuentra a sí mismo como productor en el mundo global.

\section{La alteridad cosmopolita}

"La modernización literaria latinoamericana (18701910)"18 y "José Martí en el eje de la modernización poética: Whitman, Lautréamont, Rimbaud" ${ }^{19}$ son dos ensayos en los que la modernización cultural de fin de Siglo es sinónimo de cosmopolitismo y universalidad, a través del contacto con las literaturas de la modernidad global ("Hugo, Emerson, Nietzsche, Whitman, Poe y Verlaine, Wilde, Mallarmé, Huysmans, Comte, Spencer, Renan y W. James, o Henri Bergson"20). El simbolismo, el decadentismo, el naturalismo se revelan como el "instrumental artístico" adecuado (como luego lo será el surrealismo, con su interés por lo afroamericano, y más tarde la "tendencia narrativa sureña ejemplificada por William Faulkner”) para la modernidad estética periférica, precisamente por su afinidad con "los movimientos recusatorios de la modernidad, aunque ya impregnados de las pautas contra las cuales insurgen" 21 . La atracción por la heterodoxia estética y su subjetivismo individualista -la condición de "monstruo y criminal" en que la modernidad coloca al iluminado ${ }^{22}$ - justifica, para Rama, que sólo se pueda entender a José Martí a través de un internacionalismo comparativo: Arthur Rimbaud -el "grand maudit", su experiencia del trastorno psíquico, el desdoblamiento del yo en el otro, la automatización-, Emily Dickinson, en que lee una paralela "disociación del yo y el otro” y Ralph Waldo Emerson. Dice Rama en su análisis de un apunte de Martí sobre Emerson:

En esos relámpagos estáticos se desprendía del "yo" personal y toda la conciencia era ocupada por el "otro" que en él hablaba y en ella traducía las leyes fijas del Universo, ya no vistas como verdad emanada de Dios sino como visión objetiva de su funcionamiento..$^{23}$

En la lectura comparativa de la alteridad transcultural -Martí-Rimbaud, Martí-Dickinson, Martí-Emerson- y en el universalismo despersonalizado de José Martí, que se vuelve "otro" de sí mismo en el eje transnacional, Rama no sólo contribuye a un cuestionamiento del yo triunfalista martiano, 
sino que además des-latinoamericaniza la literatura, volviéndola trans-americana y trans-nacional. El yo estético, como espacio de alteridad a-personal, implica, en Rama, la apertura a la "objetividad" comparativa del intercambio universal.

\section{La sincronización de lo asincrónico}

En el ensayo "De la concertación de los relojes atlánticos” (publicado en forma póstuma en $1984^{24}$ ), escrito como ponencia en el Congreso de Escritores dedicado a la Generación Española del 27 que se celebraría en Bogotá, Colombia, Ángel Rama realiza un análisis comparativo de las migraciones e intercambios entre los intelectuales transatlánticos, centrado alrededor de las vanguardias española y latinoamericana de los años 20. Examina el influjo del futurismo, la poesía pura, el surrealismo, el negrismo y la poesía afroantillana, la celebración de la novedad y el juvenilismo, la admiración simultánea por New York y París, por parte de las vanguardias provenientes de ambos lados del Atlántico. Pone en paralelo eventos como el homenaje a Luis de Góngora en que participaron la mayor parte de los miembros de la generación del '27 española, la Semana de Arte Moderno de 1922, el estridentismo mexicano, el martinfierrismo rioplatense, la Revista de Avance en Cuba, la Amauta peruana con Federico García Lorca, Girondo y José Ortega y Gasset. A través de esta "concertación de los relojes a un lado y otro del Atlántico" ${ }^{25}$, Rama traza un comparativismo internacionalista, cuyo hincapié en la circulación de las técnicas estéticas y en la búsqueda de la alteridad transnacional debe leerse como un intento crítico de superar los marcos nacionales y de desarmar una mismidad Latinoamericanista.
24. Ídem. "De la concertación de los relojes atlánticos". Nueva Sociedad, 1984.

25.Ibídem, p. 169. 


\section{Referencias}

ANTELO, Raúl. "Rama y la modernidad secuestrada". Estudios. Revista de investigaciones literarias y culturales. Caracas, n. 22/23, p. 17-36, 2003-2004.

APTER, Emily. Against World Literature.On the Politics of Untranslatability. New York: Verso Books, 2013.

BOURDIEU, Pierre. "Les conditions sociales de la circulation internationale des idées”. Actes de la recherche en sciences sociales. Paris, vol. 145, p. 3-8, dez. 2002.

HEIDEGGER, Martin. The Question Concerning Technology and Other Essays. New York : Garland Publishing, 1977.

HIRSCHMANN, Albert. "The Political Economy of ImportSubstitution Industrialization in Latin America”. The Quarterly Journal of Economics. Cambridge, vol. 82, n. 1, p.1-32, fev. 1968.

LEZRA, Jacques; APTER, Emily; WOOD, Michael (Dir.). Dictionary of Untranslatables: A Philosophical Lexicon. Princeton: Princeton Univ. Press, 2014.

MARX, Karl; ENGELS, Friedrich. The Communist Manifesto, 2005. Disponível em: http://www.gutenberg.org/ ebooks/61. Acesso em: 29/08/2014

MORETTI, Franco. “Conjectures on World Literature”. New Left Review. 1, p.54-68, jan/fev. 2000. . Graphs, Maps and Trees. Abstract Models for a Literary Theory. Londres: Verso, 2005.

RAMA, Ángel. "De la concertación de los relojes atlánticos”. Nueva Sociedad. n. 70, p. 163-169, 1984.

. "Founding the Latin American Literary

Community". Review: Literature and Arts of the Americas. Americas Society. vol. 15, I. 30, p. 10-13, 1981.

. "José Martí en el eje de la modernización poética:

Whitman, Lautréamont, Rimbaud”. Nueva Revista de Filología Hispánica. t. 32, n. 1, p. 96-135, 1983.

. "La modernización literaria latinoamericana (1870-

1910)”. Hispamérica. año 12, n. 36, p. 3-19, 1983.

. “La tecnificación narrativa”. Hispamérica. año 10, n. 30, p. 29-82, 1981. 
. "Literatura y cultura en América Latina”. Revista de

Crítica Literaria Latinoamericana. año 9, n.18, p. 7-35, 1983.

"Los ríos profundos, ópera de pobres". Revista

Iberoamericana. vol. 49, I. 122, p. 11-41, 1983.

.USA y los escritores latinoamericanos". Quimera.

vol. 26, p. 75-79, 1982.

SISKIND, Mariano. Cosmopolitan Desires: Global

Modernity and World Literature in LatinAmerica. Chicago:

Northwestern Univ. Press, 2014.

SPIVAK, Gayatri Chakravorty. "Planetarity”. In: LEZRA, Jacques; APTER, Emily; WOOD, Michael. Dictionary of Untranslatables: A Philosophical Lexicon. Princeton:

Princeton Univ. Press, 2014. 
